\title{
Application and evaluation about obstacle edge extraction technology in the parking assistant system
}

\author{
Feng Sang*, Chen Xingbin, Chen Shuohua, Lu Xiao \\ Faculty of Electromechanical Engineering, Guangdong University of Technology \\ No. 100 Waihuan Xi Road HEMC, Panyu District, Guangzhou, 510006, P.R China \\ Received 8 July 2011 \\ Accepted 29 November 2011
}

\begin{abstract}
The obstacle edge detection technology used in the parking assistant system helps drivers avoid obstacles, especially for inexperienced drivers. Reversing image processing, as part of smart driving, must meet the parking requirements of real-time and accuracy. Processing system should convert the color images from CCD camera to grayscale, abate noise and immunity with non-linear median filtering. And the Sobel operator that of real-time and accuracy is used in edge detection; adaptive valve segmentation technique separates these points in time for improving Hough transform identification. In order to identify the obstacles for drivers in reversing at once, it also need to be given the edge lines thicker, warning color and superimposed displayed with the original image on the terminal screen, so that the whole process can make the parking assistant system more accurate, consistent and quickly to show information.
\end{abstract}

Keywords: Parking assistant system; Obstacles edge detection; Hough transform ; Sobel operator; Subjective evaluation

In the parking assistant system, the clarity of the images not only shows a direct impact on the difficulty of reversing-drive, but also affects the safety of the vehicle in the occasion when new or inexperienced drivers cannot fully estimate the exact position of where the edge barrier during parking. If those obstacles can be displayed agreeably and accurately, it is help to improve the identification of obstacles and reduce the mental load of the driver reversing [1].

\section{Detection and treatment of obstacles edge}

Edge detection treatment is a way to extract the crossed lines between the object and the background of images with some algorithms [2]. The brief process is shown in Figure 1.

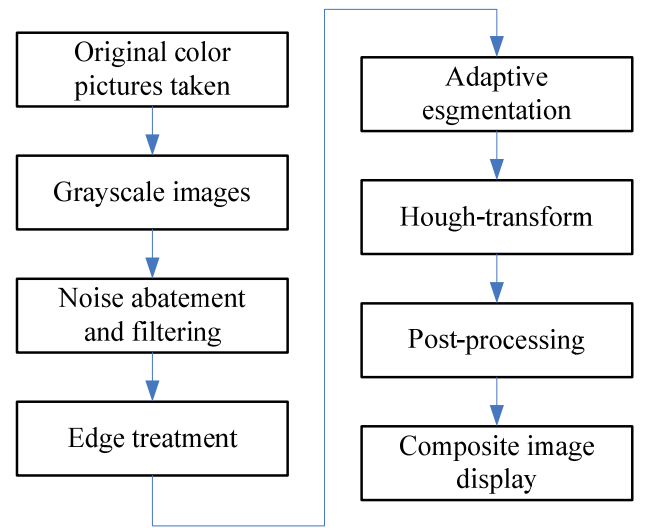

Figure1 Brief processing of reversing images

\subsection{Process of Gray-scale images}

Vast majority of the images are RGB (red, green, blue) model and three-dimensional matrix form for the space while captured by the CCD camera. But there are a lot

\footnotetext{
* Corresponding author: fengsang@gdut.edu.cn
} 
of unnecessary redundant information that had increased the workload and the time of images-processing, wasted system storage which greatly reduced the system's image display performance. Images are needed to be converted to a gray color from two-dimensional to three-dimensional to ensure to meet the premise for the edge of the identifying characteristics. Then the color images will be turned into gray-scale image, as shown in Figure 2.

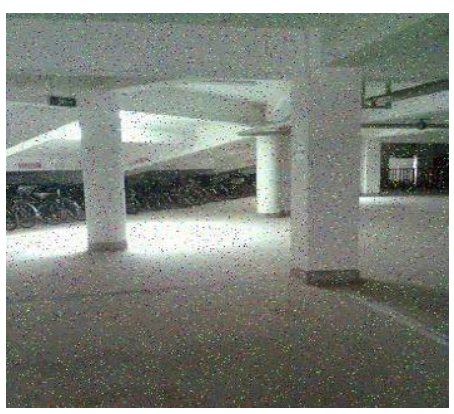

(a) color image

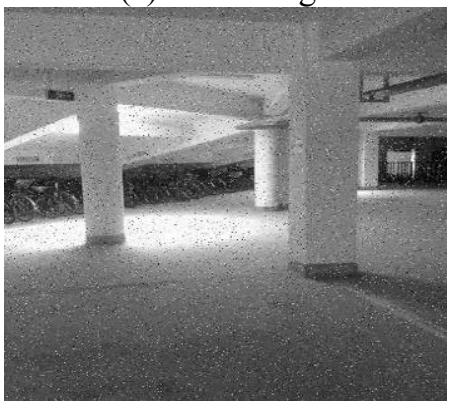

(b) gray-scale image

Figure 2 color image and gray-scale image

\subsection{Noise-abatement and filtering}

The original images are acquired on the scene with different causes of noise which will flood the signal characteristics, while severing that is not contribute to the extraction of characteristic quantities. Therefore, we must pre-process the images. The main role of image pre-processing is to remove noise interference in order to get a clearer picture. The filtering methods commonly used are Linear and Non-linear method. Linear smoothing filter has two basic ways as the neighborhood average and weighted average. Linear filter can smooth the images and remove the noise, but some of the details in the image will become blurred. For images with continuous noise, linear filter is better, such as uniform or Gaussian additive noise, however, it provides a much smoother while applied to the pulse noise. On the contrary, Non-linear method can take a better balance between the noise smooth and the images detail preservation. Finally, by comparison, Median filter as a typical non-linear method can eliminate noise in the images while keeping the details to prevent the edge blurring.

But for handling the continuous noise, median filtering could not achieve the same effect as the linear. So we set a median filter with the template to get the relatively large value of the gray pixel close to the value around the pixel, and to eliminate the pixels of isolated impulse noise perfectly. Its blur is relatively small because of not to take value on average. But its de-noising effect relates to the shape of the template or the pattern consisting with pixels which involved in computing. Square template will filter the thread and the points on the edge, and the Cross template will filter out the diagonal but retain the horizontal and vertical thin lines, while the X-shaped template is just the opposite [3]. The images after $3 \times 3,5 \times 5$ median filtering are shown in Figure 3.

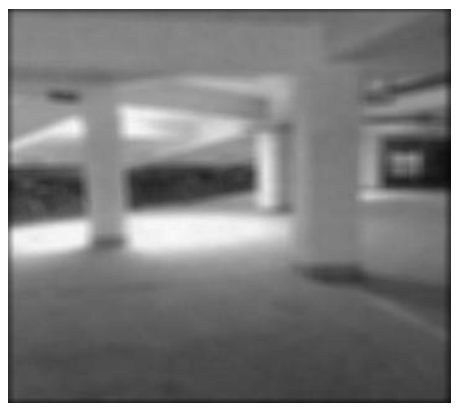

(a) $3 \times 3$ median filtering

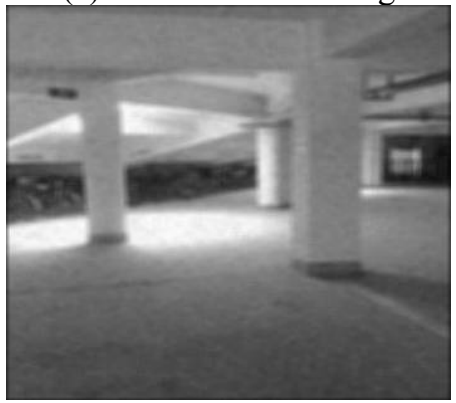

(b) $5 \times 5$ median filtering

Figure 3 images after median filtering 


\subsection{Image segmentation}

Image segmentation is divided into a number of specific features and unique nature part or subset in accordance with certain principles, and to extract the interest target. Edge detection and edge enhancement are the same as testing or enhancing an image edge through first or second order digital derivative. In the study, the edge detection has the one-degree ladder-operators such as Roberts operator, Prewitt operator, Sobel operator, and the second-order differential operators such as Laplacian operator, LOG operator, Canny operator and so on. But for the threshold segmentation, there are adaptive threshold and the best global threshold [4-7].

The traditional edge detection operators such as Prewitt, Sobel and Robert operators most are the local-window gradient operators, which are subject to certain restrictions in practical applications because they are slightly sensitive to noise. But the Canny is used to detect strong edges and weak edges with two different thresholds. The weak edge is included in the output image when it is connected to the strong edge, so this method is not to "fill" by noise but easier to detect the real weak edge than other methods.

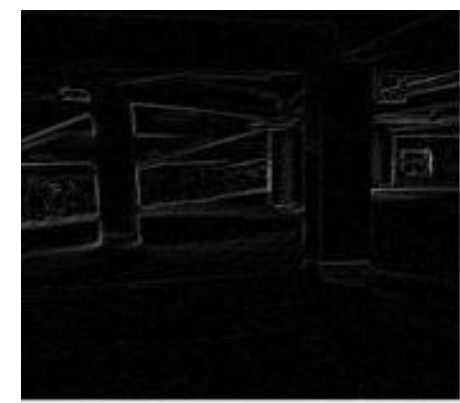

(a) with Robert operators

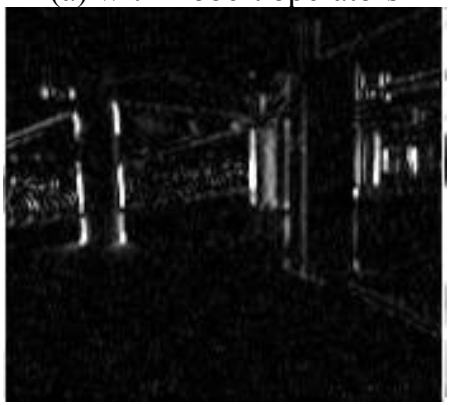

(b) with Prewitt operators

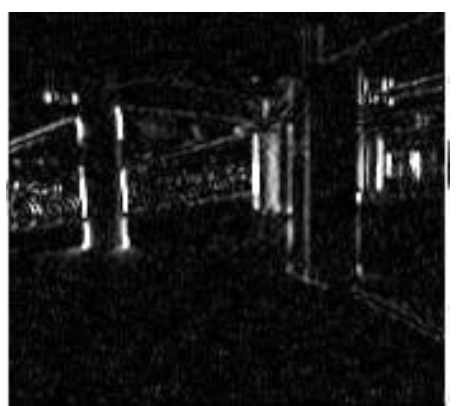

(c) with Sobel operators

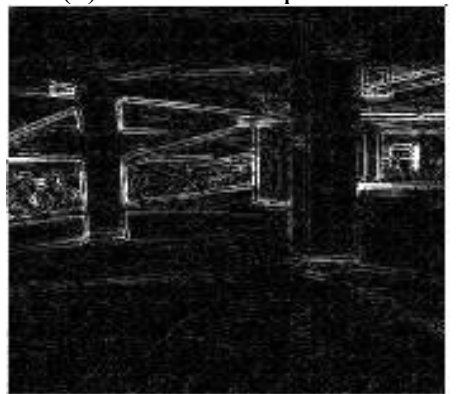

(d) with Laplacian operators

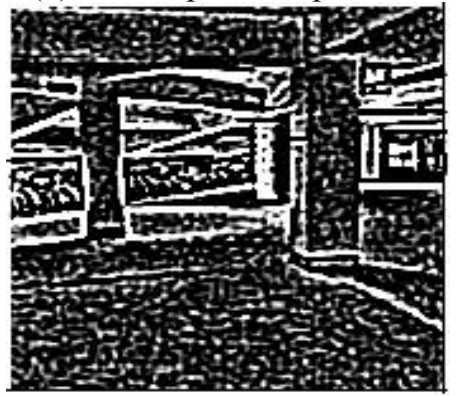

(e) with LOG operators

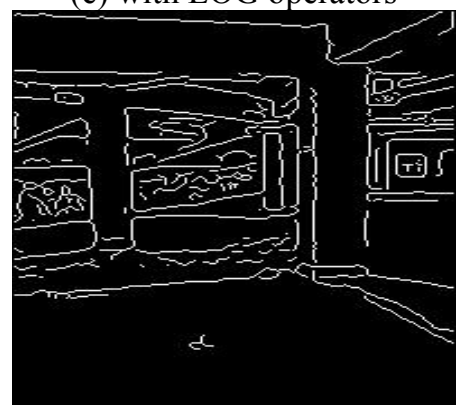

(f) with canny operators

Figure 4 Edge detection with different operator

There are three merits on the evaluation of the three indicators about edge detection performance: Good signal to noise ratio, Good positioning performance and the only response to a single edge. Canny edge detector exported with functional derivative approach is the approximation operator producing from the signal-noise ratio and the optimal positioning, which is a multi-scale 
space edge detection that basically maintained a smooth and continuous edge of the obstacle.

The results of edge detection with different operator are shown in figure 4 . The processed effect of Canny operator is the best, but it spent the longest computing time. Considering the accuracy and real-time requirements, we choose Sobel operator to detect obstacle edge.

\subsection{Edge extraction}

It is needed to detect the characteristics after image filtering, edge detection and threshold. Because the edge after the obstacle edge is not entirely a complete line after threshold and maybe a large number of unrelated isolated points or an intermittent curve[3]. So to pick out the goal we need from the obstacles edge after characterizing and selection.

Hough-transform is one of the most widely used methods, which has a strong anti-interference ability; a straight line features detection under the conditions of low signal-noise ratio. The essence of Hough-transform is how to cluster the pixels which have a certain relationship among the image space, and then find the accumulation of corresponding points in the parameter space linking with some analytical forms, that is duality [8]. The obstacle edge after being detected by Hough transform is shown in Figure 5.

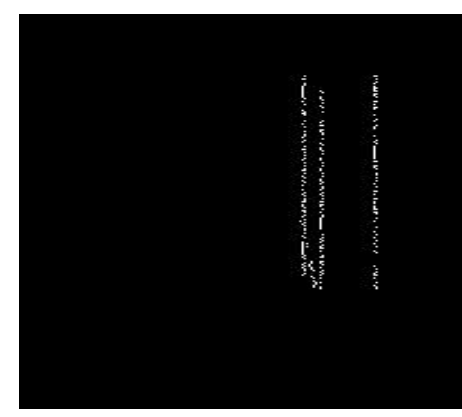

Figure 5 Image after Hough transform

\subsection{Post-processing and image synthesis}

Obstacles picture showing features as vertical, horizontal and diagonal roughly after edge detection, threshold. But the detected single pixel line cannot give purpose to remind the driver significantly. Therefore, the information marked in the obstacles accurately bases on image post-processing which mainly includes bold lines, coloration overlay and so on.
The color "Red" is used more commonly as warning color, so the results of the ultimate goal can be obtained while giving the red lines added into the original image, which is shown in Figure 6.

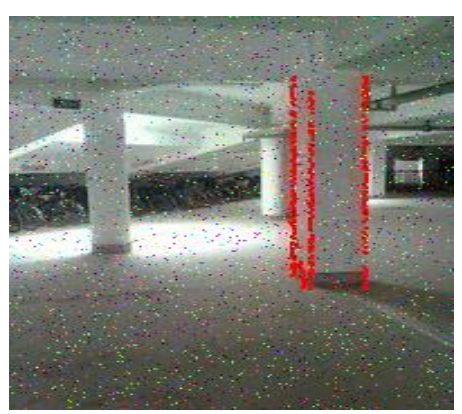

Figure 6 Edge coloration

\subsection{Special non-linear color image detection}

The way of the obstacle edge detection process above can be improved the image processing speed of the intelligent terminal in some extent, but the grayscale reflects the brightness of the image information at the expense of color information. Of course we can assign colors by some of the obstacles in post processing to meet requirements about identifying information for human. However, the edge calculated from the grayscale images does not reflect the most complex of all boundary edges information of obstacle, especially the less structured and non-linear edge of color images, its color related to highly susceptible to light strength, direction, contrast and other effects, which can easily lead to the actual edge of the image to lost in the limited calculation capacity of the intelligent terminal on-board. The detection operator in $t \alpha \beta$ Color Space chromaticity based on Sobel operator proposed by the Ruderman is to balance of the integrated approach of color images on brightness, chromaticity-difference and saturation, which also considers the allowance of the calculation of the vehicle [9].

First, the images are turned from RGB space to LMS space, and then from the LMS space to t $\alpha \beta$ space, that is to get the gradient from color changes in images, as shown in Figure 7.

From the experimental results, detection of color difference in $\alpha \beta \beta$ space can not only identify the edge information in the images rapid change brightness areas, but also detect in changes of different light areas,at the 
same time it is more sensitive to some details of the images.

So the detection of color difference in t $\alpha \beta$ space achieves similar results in remind or warning with the detection of gray-scale transformation above if without considering the calculation of the vehicle.

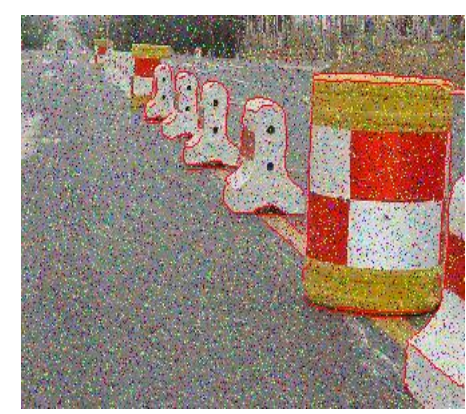

Figure 7 Edge detection in $t \alpha \beta$ space

\section{Evaluation experiment about parking images}

For the new parking assistant system in this paper, we evaluate with the methods like reaction time test and subjective evaluation. Subjective evaluation is a test to determine system performance on the direct response of subjective image quality test system of the viewers [10]. Reaction time test method refers to the evaluation of the image about the determination of the reaction time of the image viewer, and the length of time accurately [11]. Currently, it is hardly for drivers to recognize and measure accurately and rapidly through the reverse image with conventional instruments. Therefore, subjective evaluation is considered to be the most direct and relative method for image recognition, which makes an accurate characterization for the parking assistant system. But this method is not stable enough because it is often affected by objective circumstances and subjective factors or the impact of mood.

\subsection{Environment of evaluation}

Our requirements of reversing images mainly focus on assistance of reversing drive, so it is enough that the experimental conditions of subjective evaluation reach the environment of the cab. The experiment made reference to the viewing conditions proposals recommended by ITU-R.BT500 image quality subjective evaluation [12-13].

(1) Experimental ambient light brightness is lower where simulates underground parking;
(2) Display used in computer monitor is the same as that in the intelligent terminal whose peak brightness and screen brightness ratio is $\leq 0.02$;

(3) Display image size is set to 8 -inch equivalent as well as the terminal size (Resolution as $800 \times 480$ );

(4) Image-display and computer Background display fully simulate the vehicle terminal, including the color and brightness (the color ratio $\approx 0.01$ );

5) View seats should be located in the left side of the display screen about $25^{\circ} \sim 30^{\circ}$;

Major headings should be typeset in boldface with the first letter of important words capitalized.

\subsection{Images to evaluation}

We take several scenes about parking as the images of this experiment what are the static images of pillars and steps. We compare the easy-identification and real-time before and after the treatment of parking assistance system with the method how marked and colored the edge to the original images, the typical images are shown in Figure 8.

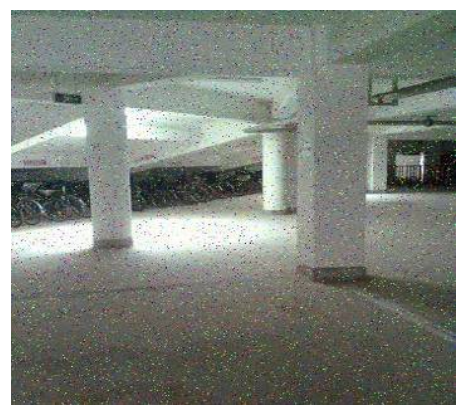

(a1) original image

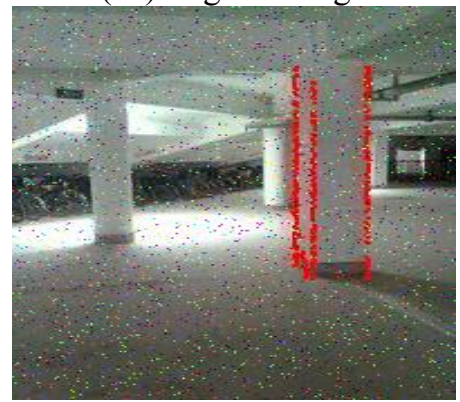

(a2) after treatment 


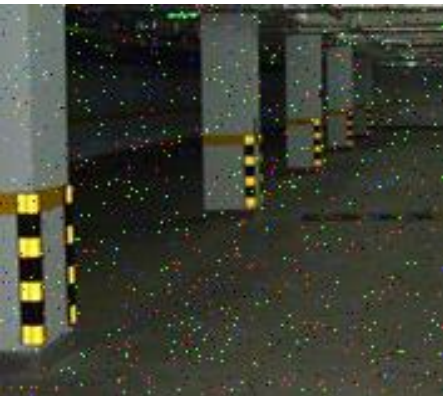

(b1) original image

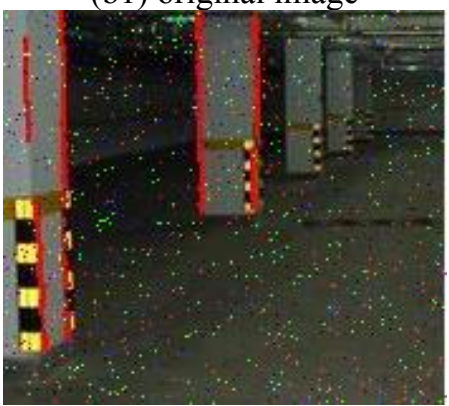

(b2) after treatment

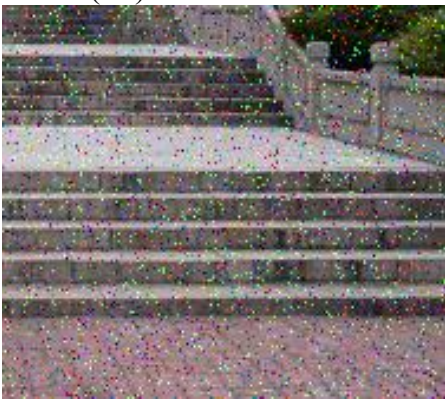

(c1) original image

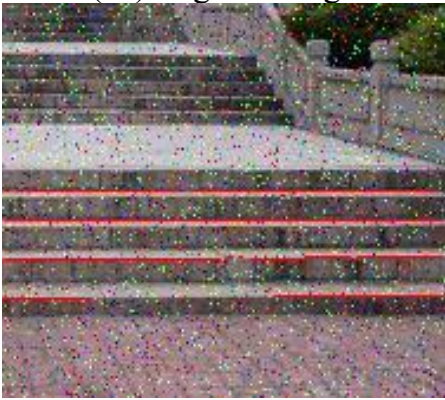

(c2) after treatment

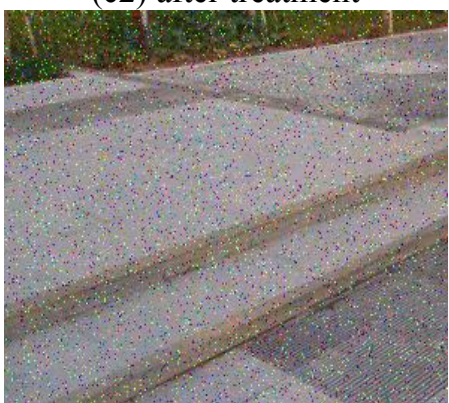

(d1) original image

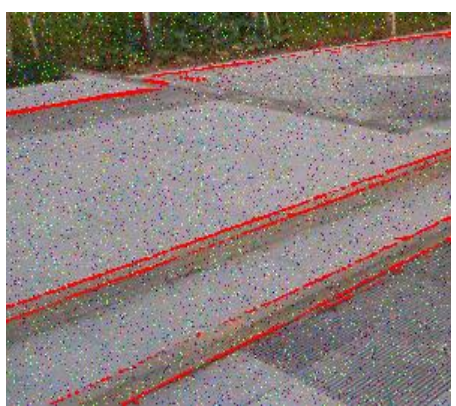

(d2) after treatment

Figure 8. Contrast images of pillars and steps before and after treatment

\subsection{Evaluators}

The parking assistance system in this paper is mainly to help beginners with no driving experience, so we take about 100 people who are non-professional groups but got the licenses and with little driving experience as evaluators. During the experiment, evaluators are needed to no strenuous exercises or no mood swings in order to reduce non-normal impacts of those factors on the test to ensure the successful completion of test.

\subsection{Grading methods}

There are many requirements for the new parking assistance system which mainly contain "obstacles to be identified quickly without confusion and observe comfortably". The reason why we take double incentive score among scorings is that it shows the main characteristic with double-stimulus display score to make the evaluate result much more stable and standardized than others.

Table 1 Grade raking

\begin{tabular}{c|l}
\hline Score & Comprehensive Evaluation \\
\hline 5 & $\begin{array}{l}\text { Great help to determine the location of obstacles, } \\
\text { reaction faster, visual fatigue-free }\end{array}$ \\
\hline 4 & $\begin{array}{l}\text { Help to determine the location of obstacles, the } \\
\text { reaction is slightly faster, slightly visual fatigue }\end{array}$ \\
\hline 3 & $\begin{array}{l}\text { No help to determine the location of obstacles. } \\
\text { Reaction no change, visual fatigue rise }\end{array}$ \\
\hline 1 & $\begin{array}{l}\text { Instead, have a negative impact to determine the } \\
\text { location of the obstacles, reaction speed decreased } \\
\text { visual fatigue easily }\end{array}$ \\
\hline
\end{tabular}


It shows for the evaluators with the reference material and the handling material at the same time in the cycle of the testing process, at last to deal with the difference between the material and reference material to measure the quality of the sub-test material. So we can compare the identification result before and after the indication by time testing and subjective ratings.

\subsection{Experiment}

It is made in random order form of slides with three grounds handle images in which inserted timer, the test time accurate to 0.01 second, icon switching and realtime recording can be achieved when evaluators simply click the button on the experimental computer. In particular, it is needed periodically in the pictures response to avoid the original image to stay between pre-handle and handled. Then the evaluators evaluate for the easy-identification whose scores reference to the ranking above. Thus, we can simply quantify the mark of real-time and accuracy image recognitions. Finally, the conclusion based on statistical analysis is used as reference for design and improvement of parking assistance system.

\section{Results analysis of subjective evaluation for parking images}

First, we should ensure the evaluation validity and rationality when we acquired large amounts of data that need to be deal with. Finally, we calculate the average score, standard deviation and the confidence interval of all the effective evaluation scores of different images according to the basic principles of statistics, as shown in table 2 .

Table 2 Results of data processing

The Recognition time (s)

\begin{tabular}{c|c|c|c|c|c}
\hline \multicolumn{2}{c|}{ Figure } & (a) & (b) & (c) & (d) \\
\hline \multirow{2}{*}{$\begin{array}{c}\text { Average } \\
\text { time }\end{array}$} & 1 original & 0.35 & 0.42 & 0.47 & 0.37 \\
\cline { 2 - 6 } & 2 after treatment & 0.14 & 0.21 & 0.23 & 0.16 \\
\hline
\end{tabular}

The score of subjective evaluation

\begin{tabular}{c|c|c|c|c}
\hline Figure & (a2) & (b2) & (c2) & (d2) \\
\hline Average score & 4.78 & 4.86 & 4.85 & 4.75 \\
\hline
\end{tabular}

Thus, subjective evaluation experiment with time reaction measurement method can be quantified and correlated statistics by the direct assessment from people, especially for drivers lack of experiences whose results have some reference value on the design criteria and optimization of parking assistance system of intelligent terminal. At the same time, we should consider different driving culture for different geographical populations on the statistical source data. So the performance of the intelligent terminals reflects the advances in technology and human-care.

\section{Conclusions and Outlooks}

This paper discusses the use of computer resources to simulate vehicle terminal to process the edge detection selected from CCD images. We make subjective evaluation with the random comparison between edge detection and calibration of several scenes, and then the data evaluated are used for average, variance, confidence or other processing requirements.

\subsection{Conclusions}

(1) The image processing about "Image cropping $\rightarrow$ Median filterin $\rightarrow$ Sobel edge detection $\rightarrow$ Thresholding $\rightarrow$ Hough transform" is indeed effective in detecting obstacles.

(2) Subjective evaluation experiment with time reaction measurement method can be quantified and correlated statistics by the direct assessment from people, and the added image evaluation correction value can overcome the subjective randomness of evaluators, all of which have some reference values on the design criteria and optimization of parking assistant system of intelligent terminal.

\subsection{Outlooks}

(1) The image processing about "Image cropping $\rightarrow$ Median filtering $\rightarrow$ Sobel edge detection $\rightarrow$ Thresholding $\rightarrow$ Hough transform" can detect the obstacle information effectively, but account for a large number of vehicle resources. So how to reduce the burden of vehicle terminal need further study.

(2) It cannot solve the problems satisfactorily with realtime and readability simply relying on resource of image processing of on-board computer. Although this paper paranoids in the image edge processing, while actually, it does not fundamentally improve the 
performance of parking assistance system, otherwise, it needs to match with CAN bus and various of alarm technologies on-board computer system.

The design of human-computer interaction about parking assistant system requires a large number of quantitative analysis through combination of subjective and objective evaluation. The solid follow-up studies are needed to gradually establish a complete evaluation system for design technical reference human-machine interface of parking assistance system.

\section{References}

1. Wuhong Wang, Fuguo Hou, Huachun Tan, Heiner Bubb, A framework for function allocation in intelligent driver interface design for comfort and safety, International Journal of Computational Intelligence Systems, 2010 3(5),531-541.

2. Chen Shuohua. Research on detecting obstacles by Machine Vision of Parking assistant System[D]. Guangdong University of technology, 2010. 05: 35 46.

3. $\mathrm{Yu}$ Jin Zhang. Image Engineering (top): image processing $[\mathrm{M}]$. Beijing: Tsinghua University Press, 2006.

4. Rui Ling Duan, QingXiang Li, Li He. Research on edge detection [J]. Optical Technology, 2005 (3): 415- 419.

5. Yang Xuan, Liang Dequn, Wan-Hai Yang. Performance analysis about Canny edge detection operator, quadratic spline wavelet operator [J]. Communications Journal, 1999 (8): 47 - 51.

6. Xiang Dong Gao, ShiSheng Huang, Ying Lin Yu. Computer Vision application in seam tracking control [J]. Control Theory and Applications, 2001 (2): 26 - 30.

7. Lin Hui, Chang Sheng Zhao, Shu Ning. Edge detection and evaluation based on Canny operator [J]. Heilongjiang Institute of Technology, 2003 (2): 3-6, 16.

8. Hough PVC, Methods and means for recognizing complex patterns[P]. USA, 3069654, 1962. (12).

9. Daniel L Ruderman. Statistics of cone responses to natural images: implications for visual coding[J]. Opt Soc Am A, 1998, 15(8): 2036-2045.

10. Zhou Wang, Alan C. Bovik. Modern Image Quality Assessment. New York: Morgan and Claypool Publishing Company, 2006.

11. J. Mannos, D. Sakrison. The effects of a visual fidelity criterion on the encoding of images. IEEE Trans. Communication, 1995, 43(12): 2959-2965.

12. ITU-R. BT500. Methodology for the subjective Assessment of the Quality of Picture, 2002.

13. ITU-T Recommendation P. 800. Methods for Subjective Determination of Transmission Quality, 1996. 